\title{
T1 Mapping for Characterization of Intracellular and Extracellular Myocardial Diseases in Heart Failure
}

\author{
Viviana Maestrini • Thomas A. Treibel • \\ Steven K. White • Marianna Fontana • James C. Moon
}

Published online: 31 July 2014

(C) The Author(s) 2014. This article is published with open access at Springerlink.com

\begin{abstract}
Heart failure (HF) is a major and growing cause of morbidity and mortality. Despite initial successes, there have been few recent therapeutic advances. A better understanding of HF pathophysiology is needed with renewed focus on the myocardium itself. A new imaging technique is now available that holds promise. T1 mapping is a cardiovascular magnetic resonance (CMR) technique for non-invasive myocardial tissue characterization. T1 alters with disease. Pre-contrast (native) $\mathrm{T} 1$ changes with a number of processes such as fibrosis, edema and infiltrations. If a post contrast scan is also done, the extracellular volume fraction (ECV) can be measured, a direct measure of the interstitium and its reciprocal, the cell volume. This dichotomy is fundamental - and now
\end{abstract}

This article is part of the Topical Collection on Heart Failure and Targeted Imaging

V. Maestrini $\cdot$ T. A. Treibel $\cdot$ S. K. White $\cdot$ M. Fontana $\cdot$

J. C. Moon $(\square)$

The Heart Hospital Imaging Centre, University College London

Hospitals, 16-18 Westmoreland Street, London W1G 8PH, UK

e-mail: j.moon@ucl.ac.uk

V. Maestrini

e-mail: viviana.maestrini@uniroma1.it

T. A. Treibel

e-mail: thomas.treibel.12@ucl.ac.uk

S. K. White

e-mail: steven.white@ucl.ac.uk

M. Fontana

e-mail:m.fontana@ucl.ac.uk

V. Maestrini

Department of Cardiovascular, Respiratory, Nephrology,

Anesthesiology \& Geriatric Sciences, Sapienza University, Rome, Italy

T. A. Treibel $\cdot$ S. K. White $\cdot$ M. Fontana $\cdot$ J. C. Moon Institute of Cardiovascular Science, University College London, London WC1E 6BT, UK measurable promising more targeted therapy and new insights into disease biology.

Keywords T1 mapping · Extracellular space $\cdot$ Heart failure · Extracellular volume fraction · Diffuse fibrosis · Intracellular space $\cdot$ Cardiac remodelling $\cdot$ Myocardial intracellular volume $\cdot$ Myocytes $\cdot$ Interstitium

\section{Introduction}

Heart failure (HF) is a complex clinical syndrome where the ability of the heart to supply physiological perfusion to organs is impaired, either by acquired or inherited disease. The key biomarker for measuring heart failure is the left ventricular ejection fraction (LVEF) - but a low ejection fraction and $\mathrm{HF}$ are not synonymous. Common causes are ischemic heart disease, hypertensive heart disease, valvular heart disease, diabetes, myocarditis, cardiomyopathies, tachycardiainduced cardiomyopathy, and systemic diseases with cardiac involvement. In most HF presentations there is more than one cause. HF represents one of the most important problems for both modern medicine and society with massive impact on individuals and a major annual cost for health systems. The annual costs for HF care exceed $\$ 40$ billions in the US, with the majority spent on hospitalizations for HF [1-3]. Moreover, hospital discharges for HF remained essentially unchanged over 10 years [4].

Damage the heart and the whole organism responds upregulated renin-angiotensin system changes, haemodynamic and skeletal muscle changes are the most well known. However, it is the myocardial response that is the most fundamental - that of the myocytes and fibroblast (the most prevalent cells in the myocardium), and extracellular space changes. These changes may be initially adaptive but may progress to be maladaptive. Common features of this 
remodelling process are cardiomyocyte hypertrophy, extracellular matrix expansion and composition alteration. Calcium handling, energy metabolism, contractile function, vasculature and cell viability may also change. These processes although deeply interwoven may combine into characteristic myocardial phenotypes which, if we can measure them, may permit us to split HF up in a subtypes, opening the door for tailored therapy according to the precise myocardial phenotype [5].

There is however a problem. There is no denying the significant advances in treatment strategies for HF, including drug and device therapy, cardiac transplantation, and mechanical circulatory support. However, despite these, the prognosis has not improved in worsening chronic HF, de novo HF and advanced or end-stage HF, which continue to have high mortality and re-admission rates [2-6]. Whilst the basic biology has pointed to a host of potential therapeutic targets with successful phase 2 trials, few of these have translated to phase 3 trial successes. There may be several reasons. Firstly, we measure the wrong thing - the ejection fraction in defining HF, despite the fact that $40 \%$ of HF has a preserved LVEF. Secondly, we group all types of HF together, thereby ignoring personalised differences in myocardial disease biology. Thirdly, our early phase trial surrogate endpoints (such as short term symptoms improving) are not well tied to important outcomes such as mortality [3]. What is needed is a better practical understanding and measurement of disease biology - fibrosis and myocyte response to split heart failure into different groups, with these biomarkers being tied to specific therapies $[2,3,7]$. This review focuses on a novel technique using standard cardiovascular magnetic (CMR) scanner, T1 mapping, to do this and its potential to act as a disease (rather than syndrome) biomarker, as a surrogate endpoint in trials and as a way of monitoring and tailoring therapy once implemented for better patient outcomes.

\section{Cardiovascular Magnetic Resonance — LGE Technique}

In the last 10 years, CMR has provided two key technologies for HF: anatomical and functional assessment using cine imaging and tissue characterization for focal abnormalities, particularly scar imaging using the late gadolinium enhancement technique (LGE). Cine imaging provided a more accurate and reproducible quantification of LVEF, size and mass, particularly in hearts that were geometrically distorted. The LGE technique for the first time allowed visualization of focal scar. When integrated, there was a step change, but perhaps not a revolution in our understanding of HF. Scar pattern, particularly in early disease provided insights into the underlying causes of myopathy, whilst the extent of scar was prognostic - and incrementally so over LVEF. The lack of scar in particular predicted functional recovery following intervention with resynchronisation (discoordination), revascularization (hibernation) or with time (stunning). Additional techniques such as T2-weighted imaging for edema and $\mathrm{T} 2 *$ imaging for iron quantification added in selected circumstances. However, a gap still remained: the LGE technique could not detect global myocardial changes such as occur in diffuse fibrosis. In addition, all scars looked the same whether there was $50 \%$ or $100 \%$ myocyte loss in a region. It is in this gap that $\mathrm{T} 1$ mapping provides new information.

\section{T1 Relaxation time and LGE Imaging}

Magnetic resonance of protons varies between tissues, depending on the macromolecular environment that water finds itself in. The $\mathrm{T} 1$ relaxation time (longitudinal relaxation time, measured in milliseconds) is a tissue-specific magnetic property and is determined by how rapidly protons re-equilibrate their spins within their environment after been excited by radiofrequency pulse. $\mathrm{T} 1$ varies with measurement technique and MRI field strength. Regional difference in T1 can be visualized by T1-weighted sequences following an intravenous bolus of extracellular contrast, gadolinium, to evaluate myocardial scar or focal fibrosis.

Gadolinium is an extracellular agent not able to enter through intact cell membrane and cleared from the blood pool after minutes. In tissue with damaged or dead cells, these kinetic effects are delayed and there is a higher accumulation of contrast due to ruptured cell membranes allowing gadolinium to passively diffuse into the cellular compartment. Gadolinium changes magnetic properties by shortening $\mathrm{T} 1$ of a tissue. If at this point, a T1-weighted inversion recovery sequence is performed, with the inversion time (TI) set manually by the operator to null "normal" remote myocardium, this will appear black and scar tissue white [8].

The LGE technique is now the gold standard test for the detection of scar across the spectrum of cardiac disease. Its assessment is reproducible and scar visualization provides a good indicator of disease etiology whilst scar extent carries prognostic information, which in many diseases is incremental to conventional prognostic markers. In HF, the LGE technique reduces diagnostic dilemmas but raises new ones: for example, it used to be a clinical conundrum that burnt out hypertrophic cardiomyopathy (HCM) could mimic dilated cardiomyopathy. The LGE technique distinguishes these with ease. However, within the HCM spectrum, whilst in early disease, the LGE pattern may point to the underlying etiology (e.g. sarcomeric protein disease, Fabrys etc.) by end stage, all the diseases start to look the same and scarred out. LGE prognostication is incrementally useful: in a large study $(n>1000)$, LGE was associated with first hospitalizations for HF after CMR, death or both across the range of ejection fractions including preserved EF. Furthermore, even if LVEF was 
severely decreased, those without LGE appeared to have less risks of hospitalization for heart failure or death [9].

However, the LGE technique has limits. Measuring the extent of LGE is difficult as different techniques produce different results, particularly in non-ischemic cardiomyopathy [10]. The technique is "black and white" — within LGE areas, complete replacement scar looks the same as $50 \%$ myocyte loss; and conversely background "normal" myocardium is all uniformly nulled meaning diffuse remote pathology and fibrosis is entirely overlooked. This latter point means the LGE technique misses global myocardial pathologies - for example LGE is an uncommon finding in pressure or volume overload disease, systemic condition with cardiac involvement, cardio-toxic effect of different agents, diabetes or hypertension cardiac effect even though all are known to have diffuse fibrosis.

Rapid technical innovations in CMR have generated a novel parametric mapping field addressing these shortcomings. These methodologies permit the routine acquisition of quantitative measure of underlying tissue-specific T1 relaxation rather than relative signal intensities.

\section{T1 Mapping}

After recent technical improvements, T1 measurement (multi-breath-hold or multiple images requiring curve fitting and processing) has been replaced by T1 mapping. In a single breath-hold, using various approaches, a T1 colour relaxation map is made [11-13]. Within the map, each given pixel value directly corresponds its underlying relaxation time that can be seen (in colour) or more formally measured, standardized, calibrated to histology $[14 \bullet \bullet, 15,16]$, compared across diseases and with normal reference ranges [17]. There are two key ways of using T1 mapping: without or before contrast (native T1 mapping); and with contrast, typically by subtracting the pre and post maps with hematocrit correction to generate the extracellular volume fraction (ECV) (Fig. 1).

\section{Native T1}

Myocardial native (non-contrast) T1 measures the T1 values from the extracellular and intracellular compartments. Measurement requires no exogenous contrast administration, making it feasible even in patients with severe kidney dysfunction. $\mathrm{T} 1$ increases with pathologies where increased water is present such as edema [18, 19] (Fig. 2e), focal or diffuse fibrosis [16] (Fig. 2f) and amyloidosis [20]. T1 reduces in the presence of lipid [21] and iron overload (Fig. 2b) [22, 23]. Using a short, single breath-hold, T1 mapping sequence to obtain native $\mathrm{T} 1$ values may complement in diseases where the LGE technique works (such as infarction) [18, 19, 24, 25] but more importantly may detect pathology otherwise missed by LGE technique, such as a pan-myocarditis [26]. In diffuse fibrosis, native $\mathrm{T} 1$ changes can be subtle. In other diseases, the changes may be very large indeed - two exemplar diseases with gross but opposite $\mathrm{T} 1$ changes are informative.

In Anderson-Fabry disease (AFD), where mutations of the $\alpha$-galactosidase gene result in intracellular lipid accumulation and left ventricular hypertrophy (LVH), the native T1 falls, and falls by many standard deviations (Fig. 2c). This fall appears to be a direct measurement of myocyte lipid storage, which had not previously been measurable. In every other cause of LVH so far explored (HCM, amyloid, aortic stenosis (AS), hypertension) $\mathrm{T} 1$ increases, meaning that a low $\mathrm{T} 1$ absolutely distinguishes AFD from all other causes of LVH with no apparent overlap [21], and with superior discrimination to other factors [27]. In AFD patients without LVH, up to half of patients have a low $\mathrm{T} 1$, suggesting that $\mathrm{T} 1$ is a marker of early cardiac involvement. One caveat of interest is that these findings are in the septum in AFD patients without heart failure. In the basal infero-lateral wall a large number of patients have an area of high $\mathrm{T} 1$ - in the area that LGE is

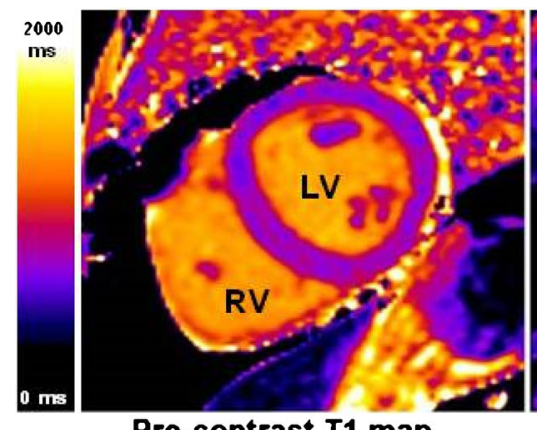

Pre-contrast T1 map

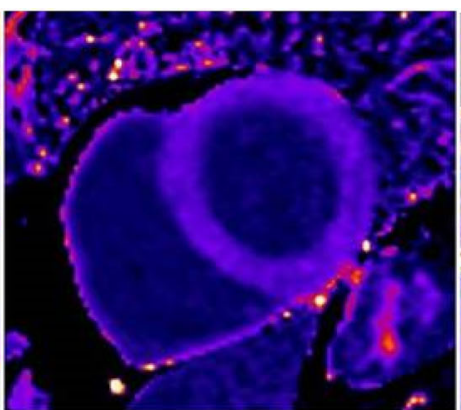

Post-contrast T1 map

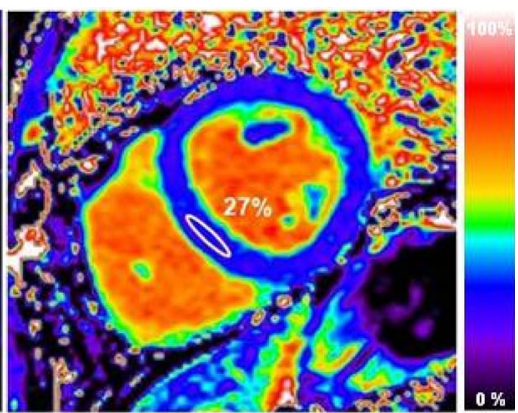

ECV map
Fig. 1 T1 maps (using MOLLI [39•], short axis view) in a healthy volunteer pre-contrast (left) and post-contrast (centre), both measured in milliseconds. For the ECV map (right), each pixel has a value of the interstitial volume as it was calculated from the two $\mathrm{T} 1$ maps. The region of interest (white) showing a normal ECV of $27 \%$ 


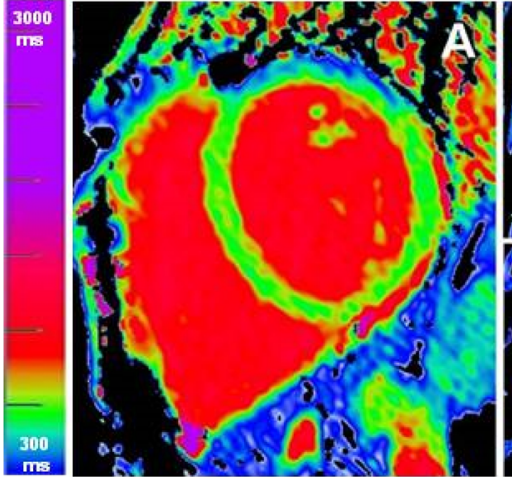

Fig. 2 Native T1 maps (using ShMOLLI [12]), all with the same colour scale. (a) healthy volunteer: the myocardium appears homogenously green and the blood is red; low T1 values (blue) from iron overload (c) and lipid storage in Fabry's disease (c) (except the infero-lateral wall

found, with a surrounding area of normal T1, suggesting a four step pathological progression from normal to low to pseudo-normalized to high T1. Whether this is true (perhaps the infero-lateral wall is never low) and whether this is a panmyocardial process leading to heart failure is currently unknown. Combined, these observations raise the possibility of T1 mapping for early diagnosis, as a surrogate endpoint in therapy trials and to monitor therapy, but much more work is needed.

In cardiac AL amyloidosis (Fig. 2d), clinically observed cardiac involvement is associated with marked elevations in native T1. These correlate with markers of LV mass, LV systolic dysfunction and markers of diastolic dysfunction (E/E' and E deceleration time). Clinically, AL amyloid cardiac involvement is classified by echocardiography and biomarkers into absent, possible and definite involvement. T1 is elevated not only in the definite cardiac involvement patients, but also in the possible and no cardiac involvement patients, at a lower level, suggesting both that T1 measurement may add value, but also that amyloid infiltration is an earlier phenomenon in this systemic disease than was previously thought [20]. The other main type of ventricular myocardial amyloid is associated with transthyretin amyloid (TTR; ATTR for amyloid from TTR). In ATTR, preliminary data detect $\mathrm{T} 1$ elevations also. These elevations are far higher than fibrotic diseases such as in AS, but appear not as high as in AL amyloid [28].

Native T1 is a feasible clinical tool. However, there are some specific hurdles to be overcome for clinical utility. Whilst pathology influences T1, so does magnet field strength and the precise approach used to its estimation [29]. These differences between approaches may be higher than the differences between health and disease pathology. Therefore at this time whilst technical developments are progressing at pace, normal reference ranges are needed for each approach and ideally for every centre. Moreover, the signal acquired is a composite signal - generated by both interstitium and myocytes. The use of an extracellular contrast agent adds another dimension to $\mathrm{T} 1$ mapping and the ability to distinguish and quantify intracellular and extracellular compartment.

\section{Extracellular Volume Fraction (ECV)}

Measuring the T1 time, following the administration of an extracellular contrast agent (gadolinium chelates), generates the possibility to dichotomize the myocardium into its cellular and extra-cellular components. In early studies, the absolute value of $\mathrm{T} 1$ post-contrast was used [30] but this has some limitations. This value is affected by renal clearance, gadolinium dose, body composition, acquisition time post bolus and hematocrit. Measuring the ratio of $\mathrm{T} 1$ changes pre and post

Table 1 T1 mapping, ECV and cardiac diseases. Summary of T1 values and ECV for different cardiac diseases

\begin{tabular}{lll}
\hline Cardiac disease & Native T1 (msec) & ECV (\%) \\
\hline Healthy volunteer & $\leftrightarrow$ & $\leftrightarrow$ \\
Severe AS & $\uparrow$ & $\uparrow$ \\
Chronic MI-LGE & $\uparrow \uparrow$ & $\uparrow \uparrow \uparrow$ \\
Chronic MI-remote & $?$ & $?$ \\
Myocarditis & $\uparrow \uparrow \uparrow$ & $\uparrow \uparrow * *$ \\
Amyloid & $\uparrow \uparrow$ & $\uparrow \uparrow$ \\
HCM-LGE & $\uparrow \uparrow$ & $\uparrow \uparrow$ \\
HCM-remote & $\uparrow$ & $\leftrightarrow *$ \\
AFD & $\downarrow \downarrow$ & $\leftrightarrow$ \\
Iron overload & $\downarrow \downarrow \downarrow$ & $?$ \\
\hline
\end{tabular}

Legend: $\leftrightarrow$ normal or $\uparrow$ increased or $\downarrow$ decreased $\mathrm{T} 1 / \mathrm{ECV}$; ? (as yet) not known

*data from the septum of AFD patients without heart failure

**data currently only in abstract form 
Fig. 3 The ECV in non-scar areas (LGE excluded) is associated with all-cause mortality even after relatively short follow-up in all-comers to a CMR service[37••]

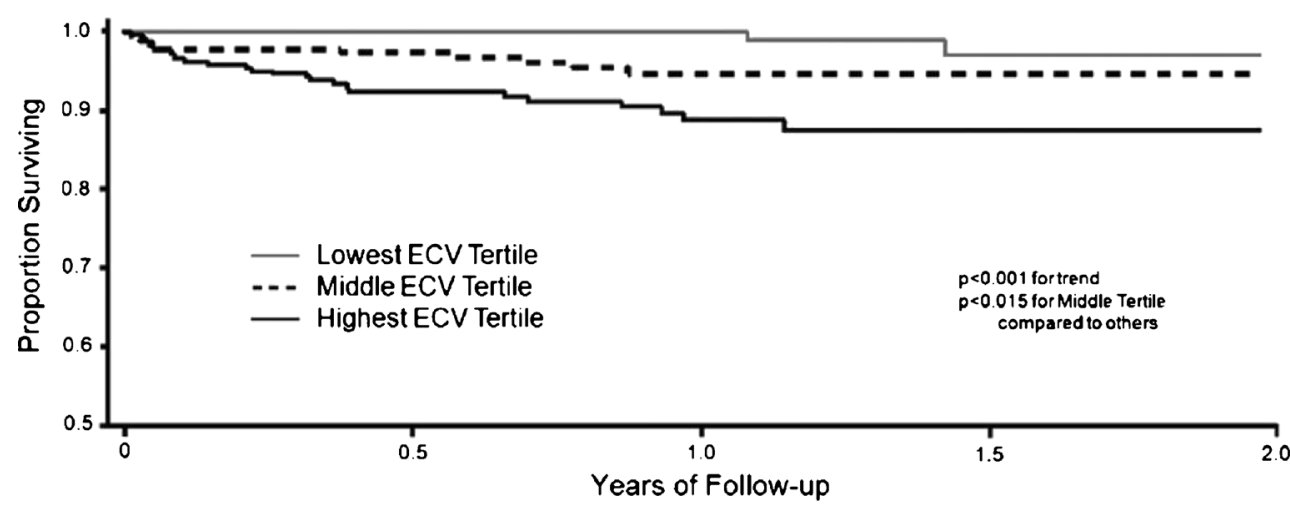

contrast administration in the myocardium and blood provides the partition coefficient, if a sufficient equilibrium of contrast was reached between blood and myocardium [31]. When corrected by hematocrit the myocardial extracellular volume (ECV) is derived [14], biologically representing the myocardial space fraction between cells; more specifically, the space between all cells, including interstitial fluid and plasma between red cells in the myocardial capillaries. Three basic methods have been used: a primed continuous infusion of contrast to reach a definitive equilibrium of distribution between plasma and interstitium; a bolus only approach with sufficient time to elapse post bolus for sufficient equilibrium; and serial time point to curve fit. Currently, it appears that the bolus only approach offers the simplest approach, but further work is needed to clarify whether any incremental benefit of the other two approaches is of sufficient merit [32, 33].

Expansion of the myocardial ECV represents a nonspecific increase in free space between cells and occurs in a variety of pathologies (Table 1). To distinguish, the degree of ECV change and the clinical context is important. Cardiac AL amyloid has a higher ECV than any other diseases generating diagnostic specificity above a certain threshold and seems to detect cardiac involvement earlier than other current tests and better than native T1 [34]. In absence of amyloid, the increased ECV value expansion is mainly due to edema or increased myocardial collagen. Edema has been little studied to date. ECV imaging can quantitatively characterize infarcted scar and atypical fibrosis, diffuse myocardial abnormalities - even when not clinically apparent on LGE images - and also the small changes occurring in myocardium with aging, even if near to detection limit $[35 \bullet \bullet, 36]$.

For low ECV expansion diseases, biases from blood pool partial volume errors need to be meticulously addressed. Nevertheless, even modest ECV changes appear prognostic. In 793 consecutive patients (excluding amyloid and HCM, measuring outside LGE areas) followed over 1 year, global ECV predicted short term-mortality (Fig. 3) [37••]. The same group also found $(\mathrm{n} \sim 1000)$ higher ECVs in diabetics associated with adverse outcome, including mortality and heart failure hospitalization. Those on renin-angiotensinaldosterone system blockade had lower ECVs. ECV also predicted mortality and/or incident hospitalization for $\mathrm{HF}$ in diabetics [38•].

The continuous technical implementation has generated automated parametric ECV maps, now available directly on the scanner, where each pixel carries directly the ECV value improving clinical utility and increasing the possibility to integrate them in clinical protocol (Fig. 4) [39•].

T1 mapping is an exciting and novel tool and transition from early development work to standardized methodologies is crucial. Differences between vendors, sequences, field strength and methodology generate confusion but also innovation, so the early standardization steps are in the form of consensus statements rather than guidelines and emphazise areas where research is needed more so than approaches not to pursue $[40 \bullet \bullet$.
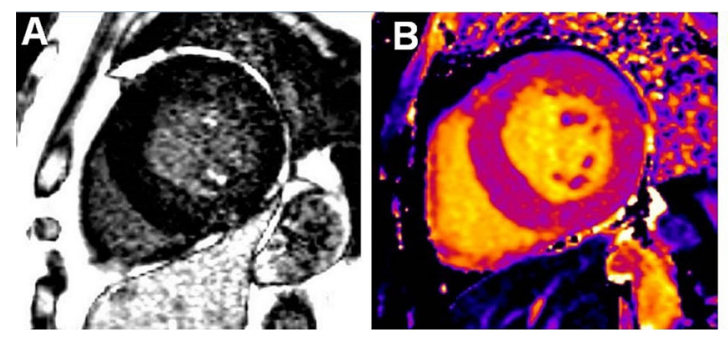

Fig. 4 A severe aortic stenosis patient with moderate concentric LVH and patchy scar on LGE imaging (a). Pre-contrast (b) and post-contrast T1 maps (c) and derived ECV map (d) add information: RV insertion point
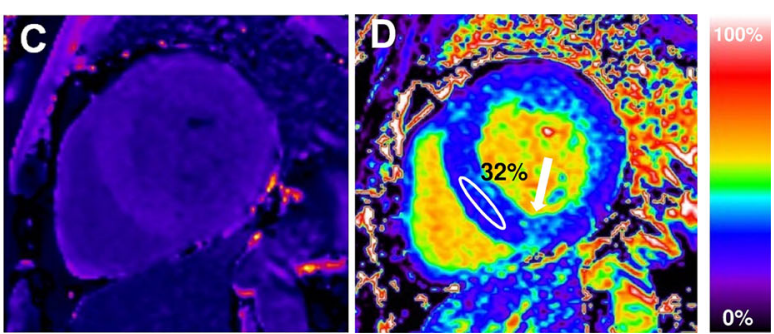

native $\mathrm{T} 1$ elevation is seen (b) and there is diffuse extracellular expansion, with an ECV of $32 \%$ 


\section{Myocardial Intracellular Volume (ICV)}

(1-ECV) represents the myocardium not accessible to the extracellular volume fraction. Accordingly, ICV represents intact myocardial cellular component proving a way to measure the cell volume. Again it is necessary to clarify that there is a bias because, even if ICV mainly represent myocytes, it also includes fibroblasts, blood cells, macrophage, etc.

ICV provides incremental information. In severe AS patients ECV has been found to be elevated but interestingly LV hypertrophy regression after valvular replacement at 6 months had no change in the ECV, but the ICV fell showing noninvasively that early $\mathrm{LVH}$ regression was cellular rather than fibrosis regression [41].

For HF, the above approaches are only now starting to be applied. The ability to measure diffuse fibrosis and partition the myocardium into cellular and extracellular compartments is promising. Specifically, it may allow to personalise therapeutic approaches and to develop new therapies targeted either to interstitial or intracellular pathways, but which may otherwise fail if early development applies them indiscriminately to an uncharacterised HF cohort. A number of studies are planned or on their way currently.

\section{Conclusions}

Drug development strategies for HF have produced few positive results over the last decade and HF continues to represent an important problem in medicine with considerable impact for health system cost. To ensure success in mortality reduction a better understanding of disease pathways is needed in-vivo. CMR has established itself as the gold standard for non-invasive myocardial tissue characterization and T1 mapping takes the technology a step further, firstly, by measuring key processes in rare diseases (iron, fat, amyloid), and secondly, by measuring diffuse fibrosis, allowing us to dichotomize the myocardium into its cellular and extra-cellular components, providing new frontiers for pathologies understanding and identifying two different therapeutic targets, cells and interstitium, with high potential impact into our understanding of HF.

\section{Compliance with Ethics Guidelines}

Conflict of Interest Viviana Maestrini, Thomas A Treibel, Steven K White, Marianna Fontana, and James C Moon declare that they have no conflict of interest.

Human and Animal Rights and Informed Consent This article does not contain any studies with human or animal subjects performed by any of the authors.
Open Access This article is distributed under the terms of the Creative Commons Attribution License which permits any use, distribution, and reproduction in any medium, provided the original author(s) and the source are credited.

\section{References}

Papers of particular interest, published in the past 3 years, have been highlighted as:

- Of importance

-. Of major importance

1. Jencks SF, Williams MV, Coleman EA. Rehospitalizations among patients in the Medicare fee-for-service program. N Engl J Med. 2009;360:1418-28.

2. Gheorghiade M, Vaduganathan M, Fonarow GC, Bonow RO. Rehospitalization for heart failure: problems and perspectives. J Am Coll Cardiol. 2013;61:391-403.

3. Butler J, Fonarow GC, Gheorghiade M. Strategies and opportunities for drug development in heart failure. JAMA: J Am Med Assoc. 2013;309:1593-4.

4. Go AS, Mozaffarian D, Roger VL, et al. Heart disease and stroke statistics-2013 update: a report from the American Heart Association. Circulation. 2013;127:e6-245.

5. Shah AM, Mann DL. In search of new therapeutic targets and strategies for heart failure: recent advances in basic science. Lancet. 2011;378:704-12.

6. Gheorghiade M, Pang PS. Acute heart failure syndromes. J Am Coll Cardiol. 2009;53:557-73.

7. Beltrami CA, Finato N, Rocco M, et al. Structural basis of end-stage failure in ischemic cardiomyopathy in humans. Circulation. 1994;89:151-63.

8. Kim RJ, Shah DJ, Judd RM. How we perform delayed enhancement imaging. J Cardiovasc Magn Reson: Off J Soc Cardiovasc Magn Reson. 2003;5:505-14.

9. Wong TC, Piehler KM, Zareba KM, et al. Myocardial damage detected by late gadolinium enhancement cardiovascular magnetic resonance is associated with subsequent hospitalization for heart failure. J Am Heart Assoc. 2013;2:e000416.

10. Flett AS, Hasleton J, Cook C, et al. Evaluation of techniques for the quantification of myocardial scar of differing etiology using cardiac magnetic resonance. J Am Coll Cardiol Img. 2011;4:150-6.

11. Messroghli DR, Greiser A, Frohlich M, et al. Optimization and validation of a fully-integrated pulse sequence for modified look-locker inversion-recovery (MOLLI) T1 mapping of the heart. J Magn Reson Imaging: JMRI. 2007;26: 1081-6.

12. Piechnik SK, Ferreira VM, Dall'Armellina E, et al. Shortened Modified Look-Locker Inversion recovery (ShMOLLI) for clinical myocardial T1-mapping at 1.5 and $3 \mathrm{~T}$ within a 9 heartbeat breathhold. J Cardiovasc Magn Reson: Off J Soc Cardiovasc Magn Reson. 2010;12:69.

13. Chow K, Flewitt JA, Green JD, et al. Saturation recovery singleshot acquisition (SASHA) for myocardial T mapping. Magn Reson Med. 2013. doi:10.1002/mrm.24878.

14.• Flett AS, Hayward MP, Ashworth MT, et al. Equilibrium contrast cardiovascular magnetic resonance for the measurement of diffuse myocardial fibrosis: preliminary validation in humans. Circulation. 2010;122:138-44. The authors describe ECV calculation and validated the technique against histology. 
15. Fontana M, White SK, Banypersad SM, et al. Comparison of T1 mapping techniques for ECV quantification. Histological validation and reproducibility of ShMOLLI versus multibreath-hold T1 quantification equilibrium contrast CMR. J Cardiovasc Magn Reson: Off J Soc Cardiovasc Magn Reson. 2012;14:88.

16. Bull S, White SK, Piechnik SK, et al. Human non-contrast T1 values and correlation with histology in diffuse fibrosis. Heart. 2013;99:932-7.

17. Sado DM, Flett AS, Banypersad SM, et al. Cardiovascular magnetic resonance measurement of myocardial extracellular volume in health and disease. Heart. 2012;98:1436-41.

18. Ferreira VM, Piechnik SK, Dall'Armellina E, et al. Non-contrast T1-mapping detects acute myocardial edema with high diagnostic accuracy: a comparison to T2-weighted cardiovascular magnetic resonance. J Cardiovasc Magn Reson: Off J Soc Cardiovasc Magn Reson. 2012;14:42.

19. Ugander M, Bagi PS, Oki AJ, et al. Myocardial edema as detected by pre-contrast $\mathrm{T} 1$ and $\mathrm{T} 2 \mathrm{CMR}$ delineates area at risk associated with acute myocardial infarction. J Am Coll Cardiol Img. 2012;5: 596-603.

20. Karamitsos TD, Piechnik SK, Banypersad SM, et al. Noncontrast T1 mapping for the diagnosis of cardiac amyloidosis. J Am Coll Cardiol Img. 2013;6:488-97.

21. Sado DM, White SK, Piechnik SK, et al. Identification and assessment of Anderson-Fabry disease by cardiovascular magnetic resonance noncontrast myocardial T1 mapping. Circ Cardiovasc Imaging. 2013;6:392-8.

22. Pedersen SF, Thrysoe SA, Robich MP, et al. Assessment of intramyocardial hemorrhage by T1-weighted cardiovascular magnetic resonance in reperfused acute myocardial infarction. $\mathrm{J}$ Cardiovasc Magn Reson: Off J Soc Cardiovasc Magn Reson. 2012;14:59.

23. Feng Y, He T, Carpenter JP, et al. In vivo comparison of myocardial $\mathrm{T} 1$ with $\mathrm{T} 2$ and $\mathrm{T} 2 *$ in thalassaemia major. J Magn Reson Imaging: JMRI. 2013;38:588-93.

24. Messroghli DR, Niendorf T, Schulz-Menger J, et al. T1 mapping in patients with acute myocardial infarction. J Cardiovasc Magn Reson: Off J Soc Cardiovasc Magn Reson. 2003;5:353-9.

25. Dall'Armellina E, Piechnik SK, Ferreira VM, et al. Cardiovascular magnetic resonance by non contrast T1-mapping allows assessment of severity of injury in acute myocardial infarction. J Cardiovasc Magn Reson: Off J Soc Cardiovasc Magn Reson. 2012;14:15.

26. Ferreira VM, Piechnik SK, Dall'armellina E, et al. T1 Mapping for the Diagnosis of Acute Myocarditis Using CMR: Comparison to T2-Weighted and Late Gadolinium Enhanced Imaging. J Am Coll Cardiol Img. 2013;6:1048-58.

27. Thompson RB, Chow K, Khan A, et al. T(1) mapping with cardiovascular MRI is highly sensitive for Fabry disease independent of hypertrophy and sex. Circ Cardiovasc Imaging. 2013;6:637-45.

28. Fontana MBS, Treibel TA, Maestrini V, et al. Myocardial T1 mapping in transthyretin amyloidosis. JACC Cardiovasc Imaging. 2013. doi:10.1016/j.jcmg.2013.10.008.

29. Raman FS, Kawel-Boehm N, Gai N, et al. Modified look-locker inversion recovery $\mathrm{T} 1$ mapping indices: assessment of accuracy and reproducibility between magnetic resonance scanners. J Cardiovasc Magn Reson: Off J Soc Cardiovasc Magn Reson. 2013;15:64.

30. Iles L, Pfluger H, Phrommintikul A, et al. Evaluation of diffuse myocardial fibrosis in heart failure with cardiac magnetic resonance contrast-enhanced T1 mapping. J Am Coll Cardiol. 2008;52:1574 80 .

31. Flacke SJ, Fischer SE, Lorenz CH. Measurement of the gadopentetate dimeglumine partition coefficient in human myocardium in vivo: normal distribution and elevation in acute and chronic infarction. Radiology. 2001;218:703-10.

32. White SK, Sado DM, Fontana M, et al. T1 mapping for myocardial extracellular volume measurement by CMR: bolus only versus primed infusion technique. J Am Coll Cardiol Img. 2013;6:955-62.

33. Schelbert EB, Testa SM, Meier CG, et al. Myocardial extravascular extracellular volume fraction measurement by gadolinium cardiovascular magnetic resonance in humans: slow infusion versus bolus. J Cardiovasc Magn Reson: Off J Soc Cardiovasc Magn Reson. 2011;13:16

34. Banypersad SM, Sado DM, Flett AS, et al. Quantification of myocardial extracellular volume fraction in systemic AL amyloidosis: an equilibrium contrast cardiovascular magnetic resonance study. Circ Cardiovase Imaging. 2013;6:34-9.

35.• Ugander M, Oki AJ, Hsu LY, et al. Extracellular volume imaging by magnetic resonance imaging provides insights into overt and subclinical myocardial pathology. Eur Heart J. 2012;33:1268-78. The authors show that ECV can quantitatively characerize MI, atypical diffuse fibrosis and subtle myocardial abnormalities not clinically apparent on LGE images.

36. Liu CY, Liu YC, Wu C, et al. Evaluation of age-related interstitial myocardial fibrosis with cardiac magnetic resonance contrastenhanced T1 mapping: MESA (Multi-Ethnic Study of Atherosclerosis). J Am Coll Cardiol. 2013;62:1280-7.

37.• Wong TC, Piehler K, Meier CG, et al. Association between extracellular matrix expansion quantified by cardiovascular magnetic resonance and short-term mortality. Circulation. 2012;126:120616. ECV may predict short-term mortality as well as composite endpoints in a large cohort of patients.

38. Wong TC, Piehler KM, Kang IA, et al. Myocardial extracellular volume fraction quantified by cardiovascular magnetic resonance is increased in diabetes and associated with mortality and incident heart failure admission. Eur Heart J. 2013. doi:10. 1093/eurheartj/eht193. ECV is associated with mortality and/or incident hospitalization for heart failure in diabetic individuals.

39. Kellman P, Wilson JR, Xue H, et al. Extracellular volume fraction mapping in the myocardium, part 1: evaluation of an automated method. J Cardiovasc Magn Reson. 2012;14:63. The authors developed a fully automated method for producing a pixel-wise ECV maps for clinical use.

40.• Moon JC, Messroghli DR, Kellman P, et al. Myocardial T1 mapping and extracellular volume quantification: a Society for Cardiovascular Magnetic Resonance (SCMR) and CMR Working Group of the European Society of Cardiology consensus statement. J Cardiovasc Magn Reson. 2013;15:92. This document provides recomendations for $T 1$ and ECV measurement, addressing issues such as scan type, scan aquisition, quality control, technical development and controversies in the field.

41. Flett AS, Sado DM, Quarta G, et al. Diffuse myocardial fibrosis in severe aortic stenosis: an equilibrium contrast cardiovascular magnetic resonance study. Eur Heart J Cardiovasc Imaging. 2012;13: 819-26. 